Rev. Elev. Méd. vét. Pays trop., 1972, 25 (2) : 309-316

\title{
Essais d'embouche intensive de taurins (Jersey x N'Dama) en Côte d'Ivoire
}

\author{
par J. L. JOUVE $\left({ }^{*}\right)$, L. LETENNEUR $\left(^{*}\right)$
}

\begin{abstract}
RESUME
Les auteurs étudiant les possibilités de croît et d'engraissement offertes par 10 bœufs métis demi-sang Jersey $\times$ N'Dama produits en Côte d'Ivoire, entretenus, en embouche intensive, en stabulation libre pendant une durée de 82 jours constatent un gain de poids journalier, après 2 mois, par animal de $750 \mathrm{~g}$ et de $593 \mathrm{~g}$ en moyenne, pour toute la durée de l'observation.

La rentabilité qui est maximale après deux mois d'embouche tend à devenir nulle après le $3^{e}$ mois.
\end{abstract}

Cette expérience a eu essentiellement pour but l'étude des possibilités de croît et d'engraissement présentées par des taurins produits en Côte d'Ivoire par croisement Jersey $\times$ N'Da$\mathrm{ma}$, entretenus en stabulation libre et recevant une alimentation comprenant à la fois du Panicum maximum et une ration composée de manioc, graines de coton et farines basses de riz.

\section{MATERIEL ET METHODES}

L'expérience se déroule à Bouaké, en saison sèche, du 19 décembre 1970 au 14 mars 1971 , soit 82 jours.

Les animaux utilisés sont des bœufs métis Jersey $\times$ N'Dama, âgés en moyenne de 37 mois et d'un poids moyen au départ de $320 \mathrm{~kg}$, au nombre de 16 , répartis par tirage au sort en deux lots.

Les 10 animaux qui constituent le lot $\mathrm{I}$, sont mis en parc et reçoivent une alimentation intensive, alors que les autres 6 animaux qui constituent le lot II, sont entretenus sur pâturage

(") I.E.M.V.T., Centre de Recherches Zootechniques de Minankro, B.P. n ${ }^{0}$ 1152, Bouake, Côte d'Ivoire. permanent avec un supplément de $1 \mathrm{~kg}$ de farine de riz par animal et par jour, pour servir de témoins de croissance.

Tous les animaux ont été régulièrement pesés en cours d'expérience.

L'état sanitaire des animaux, élevés sur la station, est connu. Ils étaient régulièrement vaccinés et traités contre les parasites gastro-intestinaux. Au moment de leur mise en parc, les animaux du lot $I$ reçoivent une injection choc d'un mélange vitaminique comprenant :

$\begin{array}{llllll}\text { Vitamine A } & . & . & . & . & 2.000 .000 \\ \text { Vitamine D U.I. } & . & . & . & . & 300.000 \text { U.I. } \\ \text { Vitamine E } & . & . & . & . & 200 \text { U.I. }\end{array}$

\section{Alimentation}

La ration prévue pour le lot I comporte les aliments ci-dessous :

Panicum maximum . . $15 \mathrm{~kg} /$ animal $/ \mathrm{j}$. Manioc . . . . $6 \mathrm{~kg} /$ animal $/ \mathrm{j}$. Graines de coton . . . $2 \mathrm{~kg} / \mathrm{animal} / \mathrm{j}$. Farines basses de riz. . $3 \mathrm{~kg} /$ animal/j.

Le modèle théorique proposé apporte les éléments suivants : 
TABLEAU $\mathrm{N}^{\circ}$ I

\begin{tabular}{|l|r|r|r|r|r|r|}
\hline Dé s i g na ti o $\mathrm{r}$ & Quantitës & M.S. kg & U.F. & M.A.D. & Ca g & P \\
\hline Panicum maximum & $15 \mathrm{~kg}$ & 3 & 1,5 & 220 & 18 & 4,5 \\
Manioc & $6 \mathrm{~kg}$ & 2,05 & 2,04 & 0 & 2,4 & 2,4 \\
Graines de coton & $2 \mathrm{~kg}$ & 1,85 & 2,06 & 250 & 3,4 & 11,4 \\
Farines de riz & $3 \mathrm{~kg}$ & 2,55 & 2,55 & 295 & 1,5 & 36 \\
\hline Total & & 9,45 & 8,15 & 765 & 25,3 & 54,3 \\
\hline Besoins & & 9,50 & 8 & 640 & 25 & 14 \\
\hline
\end{tabular}

\section{Observations}

Comme pour les expériences précédentes, elles portent sur le contrôle des consommations, l'étude des gains de poids, la surveillance sanitaire et les mesures de carcasses.

Elles sont réalisées dans les mêmes conditions.

\section{RESULTATS}

\section{Remarques générales}

Après un mois et demi d'expérience, un animal meurt accidentellement. Le lot expérimental est donc réduit à 9 têtes. Toutes les moyennes citées sont rapportées à 9 animaux.

Deux livraisons de graines de coton étaient prévues. Les graines du deuxième arrivage correspondant à une variété différente du premier sont très fibreuses et présentent une coque particulièrement dure. Elles sont délaissées par les animaux. La consommation de cet aliment ne se rétablit à un niveau convenable que pendant les deux dernières semaines de l'expérience.

Les analyses bromatologiques donnent, pour le Panicum maximum, une valeur alimentaire inférieure à celle qui avait été prévue, variant de 0,06 à $0,09 \mathrm{UF} / \mathrm{kg}$ de matière verte au lieu de 0,11 .
Le niveau de consommation des farines de riz enfin, s'est toujours trouvé inférieur au niveau théorique prévu : $3 \mathrm{~kg}$.

Ainsi, le niveau énergétique de la ration a toujours été inférieur au niveau prévu : 6,31 UF/animal/j consommés en moyenne au lieu de 8 tandis que le rapport protidique fourrager, affecté par la consommation insuffisante de graines de coton, n'a jamais dépassé 72 .

Les animaux ne se sont donc pas trouvés dans les meilleures conditions d'alimentation possibles. Aussi les croîts réalisés ne doiventils être considérés que comme l'expression partielle des aptitudes à l'embouche des bœufs métis.

\section{Résultats pondéraux}

L'évolution pondérale moyenne du lot I est très favorable pendant les deux premiers mois de l'expérience: le gain journalier de poids moyen est de $896 \mathrm{~g}$ pendant le premier mois et de $750 \mathrm{~g}$ pour l'ensemble de la période. Cette croissance diminue ensuite fortement et le gain, après 3 mois, n'est plus que de $592 \mathrm{~g} / \mathrm{j}$.

Le lot II gagne $439 \mathrm{~g} / \mathrm{j}$ pendant le premier mois, $343 \mathrm{~g} / \mathrm{j}$ après 2 mois et $206 \mathrm{~g} / \mathrm{j}$ après 3 mois.

Les résultats sont résumés dans les tableaux ci-dessous :

TABLEAU $N^{\bullet} I I$

Evolution des poids

\begin{tabular}{|c|c|c|c|c|c|}
\hline$D$ a $t$ e $s$ & $\begin{array}{l}\text { - Lot I } \\
\text { - Lot II }\end{array}$ & $\begin{array}{l}19 \text { dêcembre } \\
22 \text { décembre }\end{array}$ & $\begin{array}{l}16 \text { janvier } \\
13 \text { janvier }\end{array}$ & $\begin{array}{l}12 \text { février } \\
12 \text { février }\end{array}$ & $\begin{array}{l}11 \text { mars } \\
12 \text { mars }\end{array}$ \\
\hline & $\mathrm{N}$ & 9 & 9 & 9 & 9 \\
\hline $\begin{array}{r}\text { Lot } \\
I\end{array}$ & $\begin{array}{l}\text { Poids moyen } \\
\text { Poids pondêré }\end{array}$ & $\begin{array}{c}324,3 \pm 24,2 \\
100\end{array}$ & $\begin{array}{c}349,4 \pm 27,8 \\
107,7\end{array}$ & $\begin{array}{c}365,5 \pm 31,2 \\
112,7\end{array}$ & $\begin{array}{c}372,5 \pm 30,9 \\
114,8\end{array}$ \\
\hline & $\mathbb{N}$ & 6 & 6 & 6 & 6 \\
\hline $\begin{array}{l}\text { Lot } \\
\text { II }\end{array}$ & $\begin{array}{l}\text { Poids moyen } \\
\text { Poids pondëré }\end{array}$ & $\begin{array}{c}327,6 \pm 27,8 \\
100\end{array}$ & $\begin{array}{c}337,6 \pm 30,1 \\
103,0\end{array}$ & $\begin{array}{c}344,2 \pm 28,5 \\
105,0\end{array}$ & $\begin{array}{c}343,8 \pm 24,3 \\
104,9\end{array}$ \\
\hline
\end{tabular}


TABLEAU $N^{\circ}$ III

Evolution des gains de poids par période et croîts cumulés.

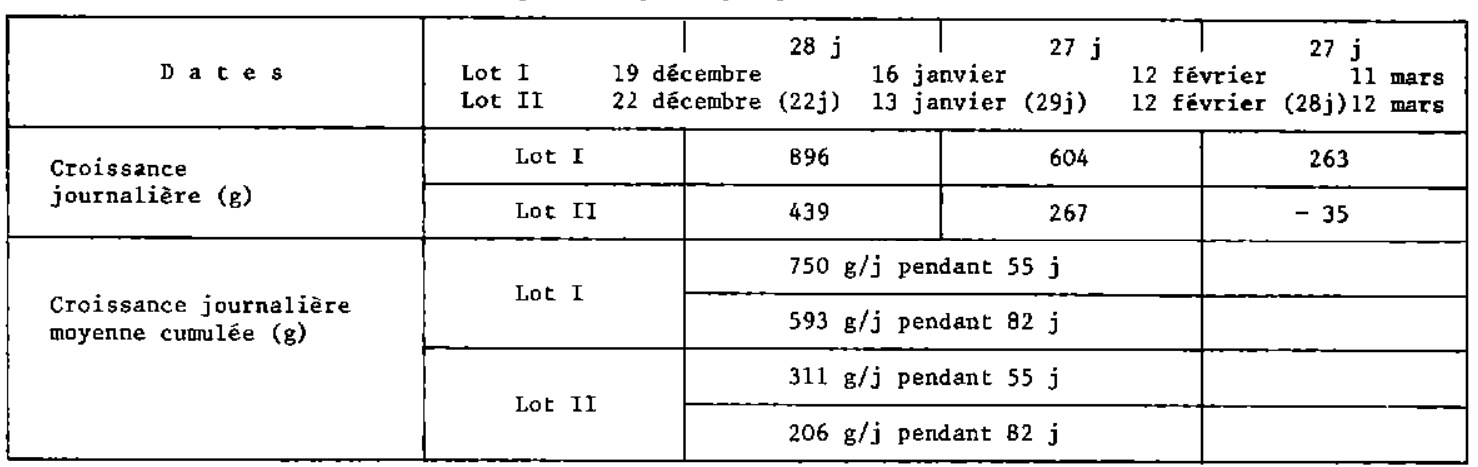

TABLEAU $\mathrm{N}^{\circ} \mathrm{IV}$

Evolution des consommations

\begin{tabular}{|c|c|c|c|c|c|}
\hline$D$ a $t$ e $s$ & $19-12$ au $16-1$ & $17-1$ au $12-2$ & $13-2$ au $11-3$ & & $\begin{array}{l}\text { Moyenne } \\
\text { Bênérale }\end{array}$ \\
\hline Panicum maximum & $12,58 \mathrm{~kg} / \mathrm{anima} / \mathrm{j}$ & $14,79 \mathrm{~kg} /$ animal $/ \mathrm{j}$ & $19,29 \mathrm{~kg} /$ animal $/ \mathrm{j}$ & 15,51 & $\mathrm{~kg} /$ animal $/ \mathrm{j}$ \\
\hline Manioc & 5,9 & 7,7 & $B, 7$ & 7,4 & $"$ \\
\hline Farines de riz & 1,8 & 2,5 & 1,7 & $2, I$ & $"$ \\
\hline Graines de coton & 1,4 & 0,4 & 0,6 & 0,8 & $"$ \\
\hline Abreuvement & $20,811 /$ animal $/ \mathrm{j}$ & $33,31 \quad 1 /$ anima $1 / \mathrm{j}$ & $25,41 \quad 1 /$ animal $/ \mathrm{j}$ & 25,51 & 1/animal/j \\
\hline
\end{tabular}

L'énergie et les matières azotées digestibles consommées par jour et par animal sont indiquées dans le tableau $\mathrm{V}$.

TABLEAU $\mathrm{N}^{\bullet} \mathrm{V}$

\begin{tabular}{|l|c|c|c|c|}
\hline \multicolumn{1}{|c|}{ D a te s } & $19-12$ au 16-1 & $17-1$ au $12-2$ & $13-2$ au 11-3 & $\begin{array}{l}\text { Moyenne } \\
\text { gênérale }\end{array}$ \\
\hline $\begin{array}{l}\text { Matières sèches moyennes } \\
\text { (kg/anima1/j) }\end{array}$ & 7,41 & 7,76 & 7,69 & 7,62 \\
$\begin{array}{l}\text { U.F. moyennes/animal/j } \\
\begin{array}{l}\text { M.A.D. moyennes } \\
\text { (g/animal/j) }\end{array}\end{array}$ & 6,11 & 6,48 & 6,35 & 6,31 \\
\hline
\end{tabular}

\section{Indices de consommation par période et indices cumulés}

L'indice de consommation varie selon les périodes. Il passe de 6,1 pendant la première semaine d'expérience à 12,7 pendant la semaine précédent l'abattage. L'indice cumulé augmente régulièrement: de 6,1 au début de l'essai il atteint la valeur de 11,3 à partir du 19 février.

La valeur élevée de l'indice en fin d'expé- rience montre que le croît obtenu est désormais surtout à base de graisse et non de muscle; l'économie des gains s'en ressent d'autant.

Les gains de poids limités et la valeur élevée de l'indice de consommation montrent que la période de finition est terminée et justifient la décision d'abattage, intervenue le 11 mars.

L'évolution des indices est représentée par le tableau VI. 
TABLEAU $\mathrm{N}^{\circ} \mathrm{VI}$

\begin{tabular}{|l|r|r|r|}
\hline \multirow{2}{*}{$\begin{array}{l}\text { Durée } \\
\text { Dates d'observations }\end{array}$} & $19-12$ & $28 \mathrm{j}$ & $27 \mathrm{j}$ \\
\hline Indices de consommation & 7,39 & 11,78 & 23,84 \\
\hline $\begin{array}{l}\text { Indices de consommation } \\
\text { cumulês }\end{array}$ & 9,35 pendant $55 \mathrm{j}$ & $12-2$ \\
\cline { 2 - 4 } & 11,47 pendant $82 \mathrm{j}$ & \\
\hline
\end{tabular}

\section{Résultats d'abattage}

Le tableau VII donne les résultats obtenus sur le lot $I$ après 3 mois ( 82 jours) d'expérience.

La conformation et l'état d'engraissement sont excellents : les profils musculaires convexes, le gras de carcasse abondant. Les rognons sont bien couverts, la graisse musculaire et la graisse de couverture toujours présentes.
A l'examen organoleptique, la viande se révèle tendre et juteuse, de qualité et de saveur comparables à une très bonne viande de France. Le rendement de carcasse est élevé, la valeur moyenne se situant à 57,79 p. 100 , le record étant de 61, 32 p. 100 . Le rendement est nettement amélioré par rapport à celui des témoins entretenus en station sur pâturage complémenté par $1 \mathrm{~kg}$ d'aliment concentré. Les meilleures performances obtenues sont indiquées dans le tableau sous la rubrique classe $\mathbf{I}$.

TABLEAU $\mathrm{N}^{\circ} \mathrm{VII}$

Résultats d'abattage

\begin{tabular}{|c|c|c|c|}
\hline & Lot I & Témoins & Classe I \\
\hline Poids avant jeûne & 372,8 & - & 412 \\
\hline Poids après $24 \mathrm{~h}$ de jeûne & 366,0 & 370,50 & 393 \\
\hline Pourcentagè de perte au jeûne & 1,82 & - & 4,61 \\
\hline Poids de la carcasse chaude & 211,6 & 203,66 & 241 \\
\hline Rendement sur poids vif & 57,79 & 54,96 & 61,32 \\
\hline Longueur de carcasse & 121,8 & 116,03 & 119,5 \\
\hline Epaisseur de la cuisse & 23,2 & 23,13 & 23,8 \\
\hline Epaisseur du plat de côte & 3,7 & 3,13 & 4,6 \\
\hline $\begin{array}{l}\text { Epaisseur de 1a cuisse en p.100 } \\
\text { de carcasse }\end{array}$ & 10,96 & 11,05 & 9,87 \\
\hline $\begin{array}{l}\text { Epaisseur de la cuisse en p.100 } \\
\text { de la longueur }\end{array}$ & 19,04 & 19,00 & 19,91 \\
\hline
\end{tabular}

Les animaux du lot II devant être utilisês pour une expérience de traction attelée n'ont pu être abattus. Les témoins citês pour les résultats d'abattage sont d'autres animaux métis entretenus au C.R.Z. dans des conditions équivalentes à celles des animaux du lot II.

Deux animaux ont pu être abattus à Bouaké. Ils ont fait l'objet de mensurations complètes dont nous donnons les résultats ci-après (tableau $\mathrm{n}^{\circ}$ VIII).

Ces chiffres confirment l'excellente conformation bouchère et le très bon état d'embonpoint des animaux.

Les morceaux de première catégorie dépassent 55 p. 100 du poids de la carcasse.
Le gras de rognon est très important, respectivement 10,5 et $15 \mathrm{~kg}$.

\section{BILAN ECONOMIQUE}

L'aspect économique de l'expérience n'a été étudié que pour le lot I soumis à une embouche intensive en parc. La comparaison économique des divers modes d'entretien fait l'objet d'une expérience particulière (étude de la naissance des taurillons N'Dama relative aux modes d'exploitation). 


\section{TABLEAU $N^{\circ}$ VIII}

\begin{tabular}{|c|c|c|}
\hline & Animal $n^{\circ} 832$ & Animal $n^{\circ} 838$ \\
\hline Poids vif aptès $24 \mathrm{~h}$ de jeûne & 357 & 346 \\
\hline Poids de la carcasse chaude & 198,5 & 193,15 \\
\hline Poids des avants & 68,5 & 67,5 \\
\hline Poids des arrıères & 118,7 & 110 \\
\hline Poids des estomacs plus intestins vides & 30,5 & 28 \\
\hline Poids du contenu digestif & 34 & 35,5 \\
\hline Poids des autres viscères & 12 & 13 \\
\hline Poids de la peau & 19 & 13 \\
\hline Poids de la tête & 20 & 20 \\
\hline Poids de la queue & 1,250 & 1,250 \\
\hline Poids des pieds & 7 & 6,5 \\
\hline Poids des rognons & 0,750 & 0,650 \\
\hline Poids du gras de rognon & 10,5 & 15 \\
\hline Longueur de carcasse & 124 & 123 \\
\hline Epaisseur de 1a cuisse & 22,5 & 23,5 \\
\hline Epaisseur du plat de côte & 3,3 & 3,6 \\
\hline Poids des arrières en p.100 de carcasse & 59,82 & 56,95 \\
\hline Poids des avants en p.100 de carcasse & 34,50 & 34,94 \\
\hline Poids du gras de rognon en p.100 de carcasse & $5,2 B$ & 7,76 \\
\hline Epaisseur de 1 a cuisse en p.100 de carcasse & 11,33 & 12,16 \\
\hline Eparsseur de la cuisse en p.100 de la longueur & 18,14 & 19,10 \\
\hline Rendement sur poids vif & 55,60 & 55,82 \\
\hline Rendement sur poids vif vide & 61,45 & 62,20 \\
\hline
\end{tabular}

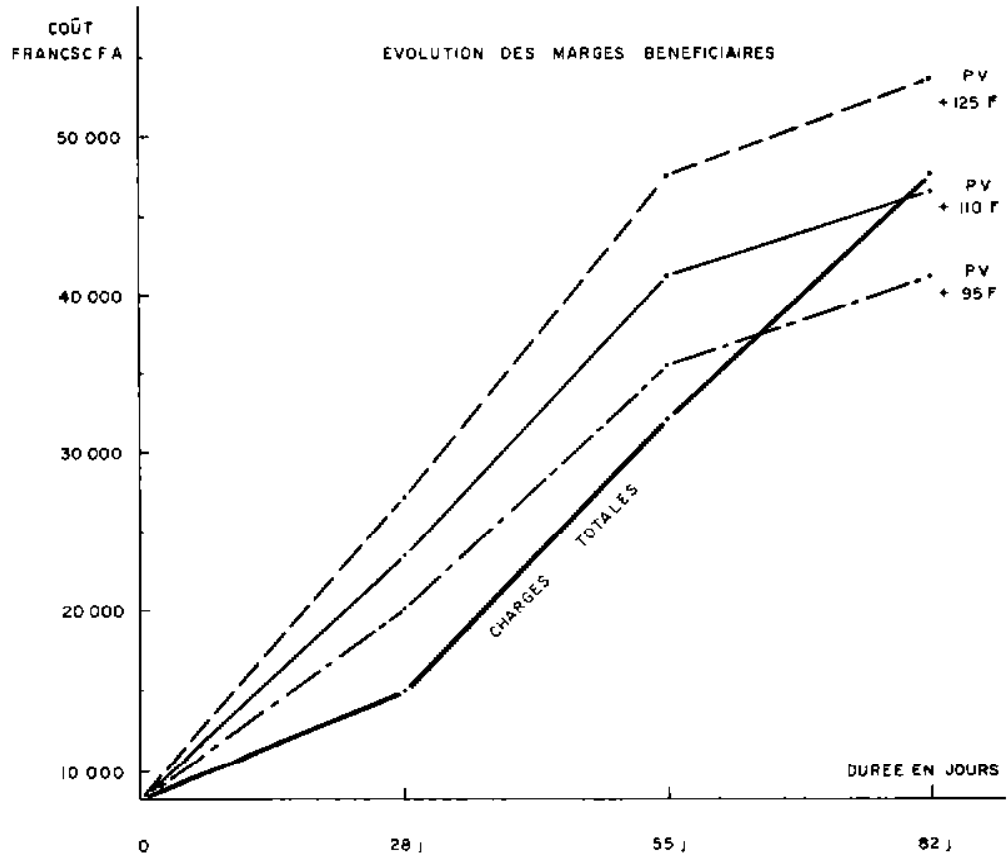


Pour le premier lot, le bilan chiffré de l'opération s'établit comme suit :

Le prix de revient des aliments utilisés est donné ci-après :
Panicum maximum

Farines de riz

Graines de coton

Manioc
5,53 F/U.F.

$6 \mathrm{~F} / \mathrm{kg}$

$3 \mathrm{~F} / \mathrm{kg}$

3 F/U.F.

TABLEAU $\mathrm{N}^{\circ} \mathrm{IX}$

\begin{tabular}{|c|c|c|c|}
\hline$N=10$ & Après 1 mois & Après 2 mois & Après 3 mois \\
\hline Poids initial du lot & 2919 & 2919 & 2919 \\
\hline${ }^{-}$Poids final & 3145 & 3292 & 3356 \\
\hline Nombre de jours & 28 & 55 & 82 \\
\hline Vâriation absolue (kg) & 226 & 375 & 437 \\
\hline Gain moyen par jour (g) & 896 & 753 & 592 \\
\hline Coût de l'alimentation. & 8575 & 20110 & $2 B 746$ \\
\hline Frais vétërinaires & 3000 & 5200 & 9500 \\
\hline Main d'oeuvre & 2160 & 4320 & 6480 \\
\hline Amortissements & 600 & 1200 & $1 B 00$ \\
\hline Intêrêt & 600 & 1200 & 1800 \\
\hline Total des charges & 14935 & 32030 & 48326 \\
\hline Prix de vente au $\mathrm{kg}$ & 125 & 125 & 125 \\
\hline Valeurs ajoutées & 28250 & $46 \quad 625$ & 54625 \\
\hline $\begin{array}{l}\text { Prix de vente - coût } \\
\text { d'alimentation }\end{array}$ & 19675 & 26515 & 25879 \\
\hline Marge nette & 13315 & 14595 & 6299 \\
\hline
\end{tabular}

A la lecture de ce tableau, on constate:

1. que les marges sont maximales à la fin du deuxième mois puis diminuent;

2. que l'expérience est rentable dans la mesure où les animaux ont été vendus sur la base de $125 \mathrm{~F}$ le $\mathrm{kg}$ vif.

Pour le même gain de poids, la rentabilité après deux mois aurait été nulle si le prix de vente avait été inférieur à $95 \mathrm{~F}$ le $\mathrm{kg}$. La rentabilité après trois mois s'annule pour un prix de vente inférieur à $110 \mathrm{~F}$ le kg.

\section{CONCLUSION}

Cette expérience d'embouche intensive d'animaux métis demi-sang Jersey-N'Dama avait pour objectif de déterminer les possibilités d'engraissement rapide d'animaux issus d'un croisement améliorateur de la race N'Dama et d'étudier les conditions de la rentabilité économique de l'opération.
L'expérience, qui a duré 82 jours du 19 décembre 1970 au 11 mars 1971 s'est terminée par l'abattage des animaux du premier lot et par un contrôle de leurs carcasses.

Les résultats généraux obtenus sont les suivants :

Lot I (10 animaux) en embouche intensive :

Poids moyen de départ $324,3 \mathrm{~kg} \pm 24,2$

Poids après 2 mois . $365,5 \mathrm{~kg} \pm 31,2$

Poids après 3 mois . $372,5 \mathrm{~kg} \pm 30,9$

Gain/j après 2 mois . $750 \mathrm{~g}$ pendant $55 \mathrm{j}$

Gain/j après 3 mois . $593 \mathrm{~g}$ pendant $82 \mathrm{j}$

L'indice de consommation moyen cumulé est de 9,3 pendant 55 jours et de 11,4 pour toute la période.

Lot II (6 animaux) (pâturage complémenté) servant de témoin de croissance:

Poids moyen de départ $327,6 \mathrm{~kg} \pm 27,8$ 
Poids après 2 mois . $344,8 \mathrm{~kg} \pm 28.5$

Poids après 3 mois . $343,8 \mathrm{~kg} \pm 24,3$

Gain/j après 2 mois . $311 \mathrm{~g}$ pendant $55 \mathrm{j}$

Gain/j après 3 mois . $206 \mathrm{~g}$ pendant $82 \mathrm{j}$

Les animaux étant au pâturage, l'indice de consommation n'a pas été évalué.

Les carcasses des animaux du lot I étaient d'excellente qualité bouchère, les rendements sur poids vif compris entre 55,60 p. 100 et 61,32 p. 100 avec une valeur moyenne de 57,79 p. 100.

L'étude du bilan économique montre que l'entreprise est rentable pour un prix de vente de $125 \mathrm{~F}$ le $\mathrm{kg}$ vif.
La rentabilité est maximale après 2 mois d'embouche. Elle tend à s'annuler après le $3^{\mathrm{e}}$ mois.

L'efficacité et l'intérêt économique des méthodes d'embouche par alimentation intensive est prouvée pour des animaux du type amélioré.

Le modèle technique proposé convient aux animaux métis. Il faut voir dans l'embouche intensive un moyen d'utiliser les produits mâles issus du croisement Jersey-N'Dama, tandis que les femelles seraient réservées à la production laitière.

La Côte d'Ivoire peut ainsi disposer à long terme d'animaux adaptés au climat et aptes à des performances zootechriques élevées.

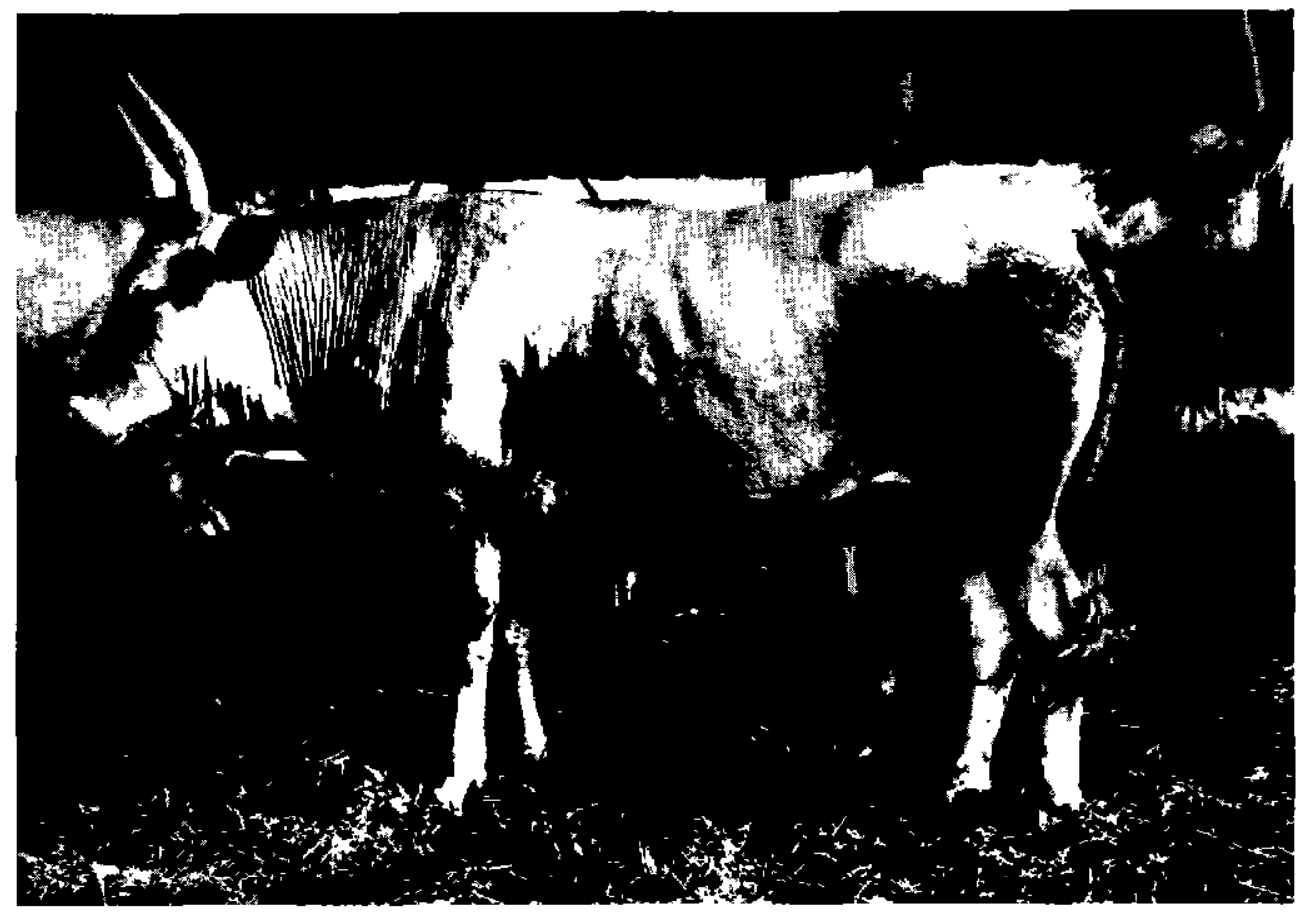

Taurin Jersey $\times$ N'Dama avant l'embouche. 


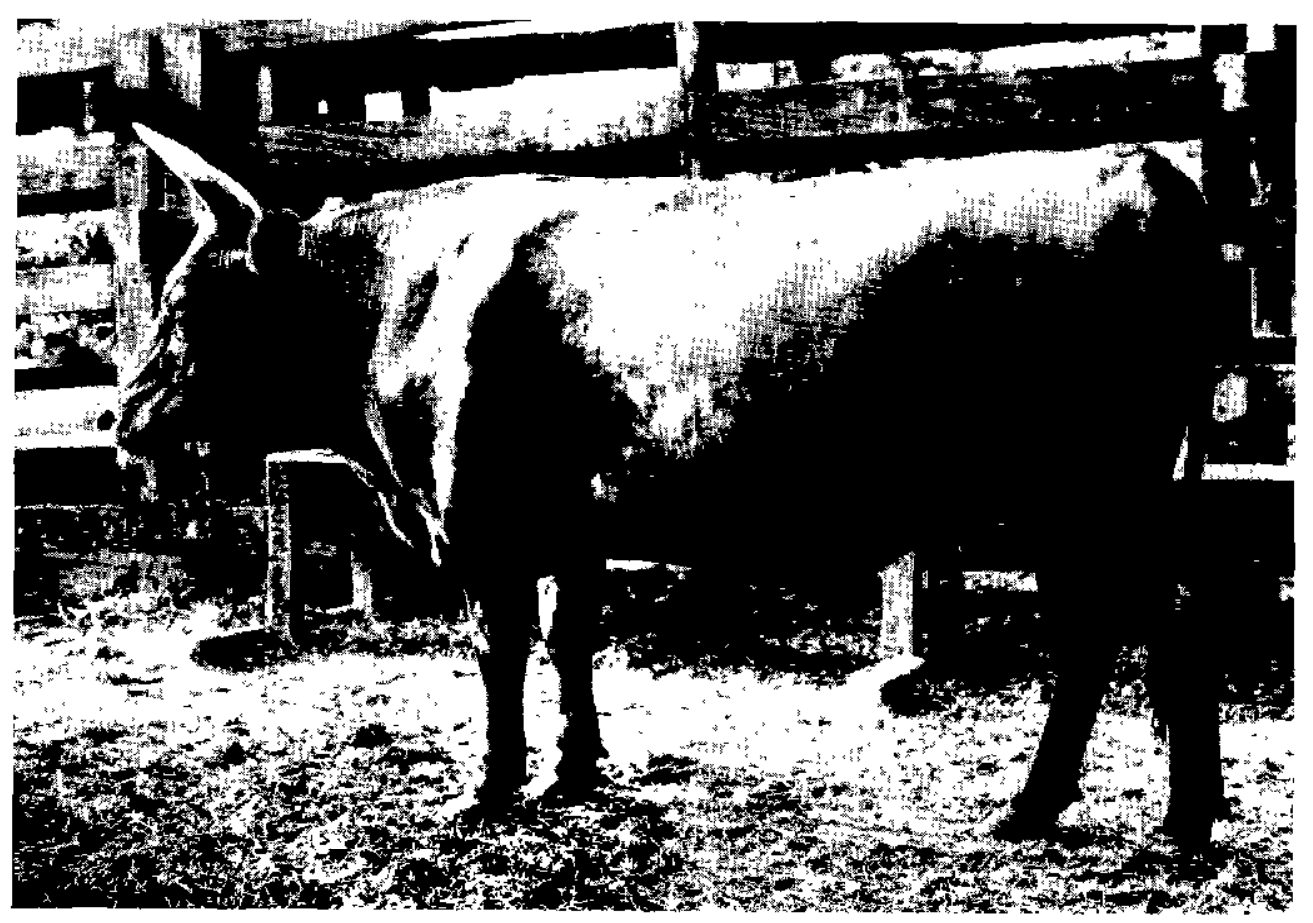

Taurin Jersey $\times$ N'Dama après embouche.

\section{SUMMARY}

Assays of intensive feeding of Jersey $\times$ N'Dama cattle in Ivory Coast

The possibilities of growth and fattening of 10 Jersey $\times$ N'Dama crossbred cattle kept in Ivory Coast are studied. Cattle, in covered pen, is given intensive feeding during 82 days. After 2 months, an average daily live weight gain of $750 \mathrm{~g}$ and $593 \mathrm{~g} /$ animal is observed during this observation.

The profit that is maximum after two months of fattening tends to become non existent after the third month.

\section{RESUMEN}

Ensayos de engorde intensivo de bovinos (Jersey $x$ N'Dama) en Costa de Marfil

Los autores estudian las posibilidades de crecimiento y de engorde de 10 bovinos mestizos media sangre Jersey $\times$ N'Dama criados en Costa de Marfil. Se mantienen dichos animales con alimentación intensiva, en libertad en establo durante 82 días.

Después de 2 meses, el aumento diario medio de peso llega a $750 \mathrm{~g}$ y a $593 \mathrm{~g}$ por animal durante el ensayo.

El beneficio que es maximo después de dos meses de engorde se hace nulo después del tercero mes. 